e0363 LEFT ANTERIOR DESCENDING ARTERY DISSECTION AND ACUTE MYOCARDIAL INFARCTION AFTER BLUNT CHEST TRAUMA: TWO CASE REPORT

doi:10.1136/hrt.2010.208967.363

${ }^{1}$ Longbin Liu, ${ }^{1}$ Hangyuan Guo, ${ }^{2}$ Haitao Lv, ${ }^{1}$ Yangbo Xin, ${ }^{1}$ Yufang Qiu, ${ }^{1}$ Fang Pen, ${ }^{1}$ Bio Yang. 'Department of Cardiology, Shaoxing People's Hospital, the First Affiliated Hospital of Shaoxing University; ' ${ }^{2}$ Wenzhou Medical College

Blunt chest trauma can cause several forms of cardiac injury. of these, coronary artery dissection and acute myocardial infarction is a rare complication. We report two cases of the left anterior descending coronary artery dissection and acute myocardial infarction after blunt chest trauma.

\section{Clinical and Research Medicine: Coronary Heart Disease \\ e0364 ASSESSMENT OF EFFECTS OF ENHANCED EXTERNAL COUNTERPULSATION ON SILENT MYOCARDIAL ISCHAEMIA AND LEFT VENTRICULAR DIASTOLIC FOUNCTION IN PATIENTS WITH CORONARY HEART DISEASE}

doi:10.1136/hrt.2010.208967.364

${ }^{1}$ Su-Hang Zhu, ${ }^{2}$ Lian-Wang Jia. ' Jinhua college of profession and techology, Jinhua, Zhejiang, China; ${ }^{2}$ Jinhua central hospital, Jinhua, Zhejiang, China

Objective To assess the efficiency of enhanced external counterpulsation (EECP)on silent myocardial ischaemic (SMI)and left ventricular diastolic function (LVDF) in patients with coronary heart disease.

Methods 78 cases of coronary heart disease were randomly into group A (39 cases) and group (39 cases). The group B was treated with normal treatment aspirin, isosorbide mononitrate metoprolol and pavasatatin, the group A was treated with EECP based on the normal treatment for 4 weeks. The changes of myocardial ischaemia and cardiac function were observed by DynamicECG and Doppller echocardiograpy combination with tissue Doppler imaging (TDI) before and after treatment.

Results Frequency and sustained time of ST segment depression, and total loading of myocardial ischaemia were decreased significantly in two groups ( $p<0.01$ respectively) after treatment, but it decreased more significantly $(p<0.01)$ in group $A$ than in routine group $B$. There was no obvious difference $(p>0.05)$ on the left ventricular systolic function $(\mathrm{CO}, \mathrm{EF})$ between the two groups after treatment. LVDF was reduced in patients of two groups before treatment. After treatment indexes of LVDF (E, A, E/A, DC, Ea, Aa, Ea/Aa, E/Ea) were improved (all $\mathrm{p}<0.01$ ) in the group $\mathrm{A}$, while there were different $(p<0.05)$ in group $B$ before and after treatment. However LVDF in group A was better $(p<0.01)$ than that of group B. No sever adverse effects found in treating with EECP.

Conclusion There has obvious therapeutic efficacy of EECP on SMI and LVDF in patients with coronary heart disease.

\section{e0365 CLINICAL STUDY ON RELATIONSHIP BETWEEN SERUM GAMMA-GLUTAMYLTRANSFERASE AND CORONARY HEART DISEASE IN WOMEN}

doi:10.1136/hrt.2010.208967.365

Song Jing, Wei Yidong, Fu Yuanyuan, Wang Yong, Hou Lei, Xu Yawei. Department of Cardiology Shanghai Tenth People's Hospital Tongji University

Objective To study the relationship between serum gamma-glutamyltransferase (GGT) and coronary heart disease (CHD) in women. Methods 636 patients (354 men, 282 women) undergoing coronary angiography were enrolled from March 2009 to November 2009 in the department of cardiology, Shanghai Tenth People's Hospital, Tongji University. The various indicators of blood testing and clinical data were collected among 636 patients. According to the results of coronary angiography, men and women respectively divided into $\mathrm{CHD}$ group and non-CHD group. According to the level of serum GGT, men and women respectively divided into five grade groups (normal low, normal high, moderately elevated, elevated, highly elevated).

Results The serum GGT levels in CHD group were statistically different from those in non-CHD group in women $(p=0.010)$ but not in men $(p=0.480)$. More women had elevated serum GGT levels in CHD group than those in non-CHD group $(p=0.009, O R=1.877)$, but this significant difference was not found in men $(p=0.427$, $\mathrm{OR}=0.829$ ). The prevalence of $\mathrm{CHD}$ in women was significantly increased as serum GGT levels elevated $(p=0.012)$, but this trend was not found in men $(p=0.369)$. The levels of alanine aminotransferase (ALT), aspartate aminotransferase (AST), direct bilirubin (DBIL), post prandial blood glucose (PBG) and the values of Ln triglycerides (LnTG) in women were positively correlated with the values of Ln serum GGT levels (LnGGT) separately, but levels of high density lipoprotein cholesterol (HDL-C) were negatively correlated with LnGGT. The levels of ALT, uric acid (UA), fasting blood glucose (FBG), total cholesterol (TC) and LnTG in men were positively correlated with the values LnGGT separately. Logistic regression indicated that serum GGT was independent risk factor of CHD in women (OR=1.782, 95\% CI 1.043 to 3.043, $\mathrm{p}=0.034$ ).

Conclusion (1) Serum GGT was correlated with morbidity of CHD in women. (2) The prevalence of CHD in women was significantly increased as serum GGT levels elevated. (3) The values of LnGGT were positively correlated with the levels of PBG and the values of LnTG in women, but negatively correlated with the levels of HDLC. (4) Serum GGT was independent risk factor of CHD in women.

\section{e0366 EFFECT OF THE COMPOSITIE SALVIAE DROPPING PILL ON BLOOD LIPIDS AND LARGE ARTERY ELASTICITY IN PATIENTS WITH CORONARY HEART DISEASE OR CORONARY RISK FACTORS}

doi:10.1136/hrt.2010.208967.366

Xiangping Li, Shuiping Zhao, Yingnan Qin. Department of Cardiology, The Second Xiangya Hospital, Central South University, Changsha Hunan

Objectives To investigate the effect of the compositie salviae dropping pill on blood lipids and large artery elasticity in patients with coronary heart disease (CHD) or coronary risk factors.

Methods 63 patients with $\mathrm{CHD}$ or coronary risk factors were randomly divided into two groups: trial group $(n=32)$, received compositie salviae dropping pill 10 pill three times a day in addition to routine therapy; control group $(n=31)$, treated with routine therapy. All subjects underwent laboratory measurements including the concentrations of serum total cholesterol (TC), triglyceride (TG), low density lipoprotein cholesterol (LDL-C), high density lipoprotein (HDL-C), fast blood sugar (FBS), and high sensitivity C-reactive protein (hs-CRP) before and 4, 24 weeks after the treatment. Carotid-femoral PWV (cfPWV), carotid-radial PWV (crPWV) and femoral-ankle PWV (faPWV) in both groups were measured by PP-1000 automatic PWV measuring system before and after the treatment.

Results There were no significant difference in the baseline parameters between two groups (all $\mathrm{p}>0.05$ ). After treatment for 24 weeks, there was a notable decrease of TG and hs-CRP levels in both two groups $(\mathrm{p}<0.05$ or 0.01$)$ and TC and LDL-C levels in trial group $(p<0.01)$. After 24 weeks, the LDL-C level was lower in trial group than in control group $(p<0.01) .24$ weeks later, cfPWV and faPWV were significantly lower in trial group than baseline and in 